\title{
General Critical Properties of the Dynamics of Scientific Discovery
}

\author{
Luís M. A. Bettencourt ${ }^{1,2}$ and David I. Kaiser ${ }^{3}$ \\ 1 Theoretical Division, Los Alamos National Laboratory, Los Alamos NM, USA. \\ ${ }^{2}$ Santa Fe Institute, 1399 Hyde Park Road, Santa Fe NM 87501 U.S.A. \\ 3 Program in Science, Technology, and Society and Center for Theoretical Physics, \\ Massachusetts Institute of Technology, 77 Massachusetts Avenue, Cambridge, MA 02139 U.S.A.
}

\begin{abstract}
Scicntific fields are difficult to define and compare, yet there is a general sense that they undergo similar stages of development. From this point of view it becomes important to determine if these superficial similarities can be translated into a general framework that would quantify the general advent and subsequent dynamics of scientific ideas. Such a framework would have important practical applications of allowing us to compare fields that superficially may appear different, in terms of their subject matter, research techniques, typical collaboration size, etc. Particularly important in a field's history is the moment at which conceptual and technical unification allows widespread exchange of ideas and collaboration, at which point networks of collaboration show the analog of a percolation phenomenon, developing a giant connected component containing most authors. Here we investigate the generality of this topological transition in the collaboration structure of scientific fields as they grow and become denser. We develop a general theoretical framework in which each scientific field is an instantiation of the same large-scale topological critical phenomenon. We consider whether the evidence from a variety of specific fields is consistent with this picture, and estimate critical exponents associated with the transition. Wo then discuss the generality of the phenomenon and to what extent we may expect other scientific fields - including very large ones - to follow the same dynamics.
\end{abstract}

Keywords: Evolution of Science - Complex Networks Critical Phenomena - Discovery - Topological Transition

\section{Introduction}

The evolution of science and technology is a subject of enormous intellectual and societal importance. One inescapable feature has been the explosion of scientific publishing in recent decades, along with the enormous growth in the indexing and availability of scholarly 
documents. These developments open new opportunities to construct a robust, quantitative understanding of the processes by which scientific and technical fields emerge and grow. A central question to these studies is whether there are general structures and dynamics in the evolution of science that unify the development of different fields across disciplines and time. There are certainly a variety of emerging methodologies that unify the study of science. These include population dynamical models $[1,2,3,4,5,6,7]$, networks of cocitation $[8,9]$ and collaboration $[10,11,12,13,14,15,16,17]$, disciplinary maps of science $[18,19,20,21]$ and phylogenetic term analyses [22, 23], among others. But a more profound question is whether studies using these methodologies and others capture common dynamics across fields. Moreover, if such general frameworks exist, what insights for society and policy might these common dynamics reveal?

The concept of a general science of science and technology is an old multidisciplinary question. Foundational work [24, 25] certainly assumed that different fields reflect, at least in some general sense, a common dynamics of discovery. Analysts like Kuhn [24] hypothesized a general structure by which scientific communities coalesced, evaluated potential anomalies or crises, and perhaps dissolved. Yet it is also evident that there are many differences of time scales, level of investment, necessary equipment, collaboration size and scientific productivity that distinguish different fields of science, as standard measures of scientific impact have started to show $[9,26]$. In the face of these more recent measures that emphasize distinctions among scientific fields and differences in their evolution over time, do any commonly shared features remain?

The availability of new data has made the mapping of scientific fields increasingly possible, but we believe that a return to the question of what is common across scientific fields is now important and timely. Here we develop an integrated analysis of several fields of science, spanning several disciplines, methods, and sizes, from theoretical physics to computer science and biomedical research. We show that there exists a strong set of commonalities across all these fields, signaling the processes of discovery and invention and the advent of a unified 
community of practice. These changes in social dynamics and cognitive content are manifest in terms of a topological transformation of their large-scale structure of collaboration. Moreover, we go one step further to show how all fields analyzed here can be interpreted as undergoing the same kind of idealized phase transition, though certain dynamical and small-scale structural aspects of each field vary.

The main methodological challenge in comparing different fields from this perspective is the enormous variation in their size (measured in terms of number of publications and authors), which is manifested also in their network and dynamical properties. By any measure it is common to find fields with only tens or hundreds of authors, which are characterized by clear unifying concepts and techniques. However, we often also refer to much larger fields with hundreds of thousands of authors or more, which show much more diversity and are much more loosely connected. Are these instances of the same phenomenon? To address these issues we develop a theoretical framework based on familiar ideas of universality in critical phenomena observed in finite-size systems. We show that within such a framework, any field can be thought of as the realization of certain general dynamics of agglomeration and percolation. Moreover, we demonstrate that in a very specific quantitative sense, all fields are comparable under scale transformations and map to an idealized dynamical social network critical phenomenon that can exist at any scale, including the limit of very large numbers of authors.

\section{Results}

\subsection{Characterizing Growth Over Time}

In this study we have examined the growth and development of several scientific and technical fields as they changed over a time-scale of decades. The fields vary greatly in size and composition: from relatively modest-sized communities in theoretical physics such as cosmic strings or cosmological inflation, in which authors have similar training; to benchtop biomedical topics like research on scrapie and prions, which incorporate co-authors of varied 
expertise who work together on a narrowly-defined problem; to huge interdisciplinary fields like nanotechnology and sustainability science, which feature authors from a wide range of specialties. Whereas we have examined other aspects of the growth of several of these fields in previous work [7, 17], the two largest fields in our sample — sustainability science and nanotechnology — represent brand-new datasets. ${ }^{1}$ See Table 1.

$\begin{array}{cccc}\text { field } & \text { years } & \text { No.publications } & \text { No. authors } \\ \text { cosmic strings } & 1976-2005 & 2,443 & 2,292 \\ \text { inflation } & 1981-2005 & 5,135 & 3,410 \\ \text { quantum computing } & 1967-2005 & 8,946 & 7,518 \\ \text { scrapie and prions } & 1960-2005 & 11,074 & 14,620 \\ \text { string theory } & 1974-2005 & 9,766 & 25,022 \\ \text { sustainability science } & 1974-2009 & 20,455 & 36,984 \\ \text { nanotechnology } & 1990-2010 & 521,075 & 333,990\end{array}$

Table 1. Scientific fields included in our study.

In our previous work we have found two features of scientific authorship that can be used to simplify our analysis. First, as our "mean-field" population-modeling has shown, the total number of authors (nodes), $N(t)$, plays the role of the relevant time-like dynamical variable [7]. For example, we found that the total number of articles grows as a simple power-law of the total number of authors, even as both quantities display more complicated growth patterns when measured with respect to time, $t$. Thus we may adopt $N(t)$, the number of authors in a given field at time $t$, as our basic time-like variable, akin to the kinds of rescaling commonly adopted in cosmological models such as the universal scale factor, $a(t)$, or conformal time, $\eta=\int d t / a(t)$.

Second, in our previous study of collaboration networks [17], we found a simple scaling relationship between the number of co-authorship links (or edges) per node, $E(t)$, and the number of authors (nodes), $N(t)$,

$$
E(t)=E_{i}[N(t)]^{\alpha_{i}}
$$

\footnotetext{
${ }^{1}$ On the search strategies used to construct the datasets for the first five fields listed in Table 1, see the appendix in [7]. To construct the database of all authors and articles in nanotechnology, we implemented the search strategy described in [27] within the Web of Science database. The database of authors and articles on sustainability science was generated for a recent study of the evolution and structure of this field, see [28].
} 
where $E_{i}$ and $\alpha_{i}$ are constants, different for each scientific field, $i$, under consideration. The quantity $\alpha$ is often termed the "densification exponent": networks with $\alpha>1$ show increasing numbers of links per node as the network itself grows over time. Fields with $\alpha>1$, in other words, grow more dense as they evolve [29]. Empirically, virtually every field we studied had an exponent, $\alpha>1$, with most falling in the range $\alpha=[1.05,1.38]$; only cold fusion displayed a scaling exponent consistent with 1 [17]. In our latest studies, we have found the same behavior for much larger scientific fields: both for sustainability science, a field that has included approximately 37,000 authors over the past few decades and has a densification exponent of $\alpha=1.27$; and for nanotechnology, a field that has included more than 330,000 authors over the past twenty years and has a densification exponent of $\alpha=1.36$. (See Figure 1, below.)

Such densification over time provides one simple example of the clarity that can come from considering $N$ to be the time-like variable, rather than ordinary time, $t$. Consider, for example, the plots in Figure 1. Panels A and B show the number of new authors per year in nanotechnology and sustainability science, respectively. For nanotechnology, new authors per year grew roughly linearly over much of the time period of interest, whereas new authors per year in sustainability science grew exponentially. Yet when plotting the number of links in each network, $E(t)$, versus number of authors, $N(t)$, as in panels $\mathrm{C}$ and $\mathrm{D}$, both fields betray the simple scaling behavior of Eq. (1) - a simplicity of structure that is not at all apparent from graphs like those in panels A and B.

\subsection{From Densification to Percolation}

A quantity of interest in any network study is the degree of a node, $k$, which counts the number of links connected to that node. Given the form of Eq. (1), the average degree, $\langle k\rangle$, should obey a simple scaling relation with network size, $N$, of the form

$$
\langle k\rangle=\frac{E(t)}{N(t)}=E_{i}[N(t)]^{\alpha_{i}-1}
$$



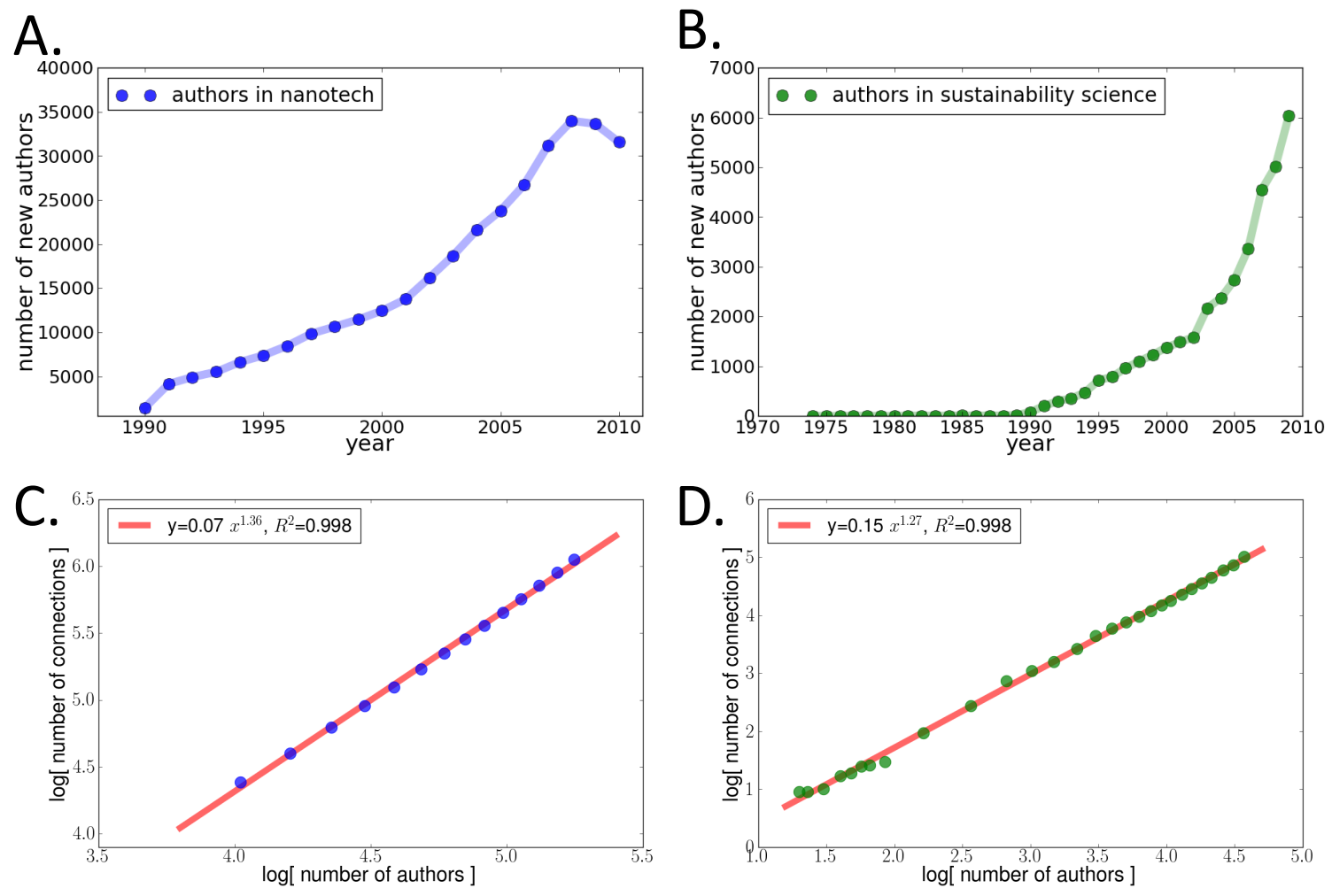

Figure 1: Time evolution of fields of science and the densification of collaboration networks. A. The number of new authors per year in nanotechnology vs. time. B. The number of new authors per year in sustainability science vs. time. Regardless of the details of the temporal evolution of the field, the number of collaboration (co-authorship) links increases in a scaleinvariant way with the number of authors in the network, as shown in $\mathrm{C}$ for nanotechnology and D for sustainability science. These patterns are similar to those we have found in [17] for a variety of smaller scientific fields. 
In [17] we found that fields with exponents $\alpha>1$ underwent a topological transition, akin to percolation: at some finite time in each field's evolution, one giant connected cluster emerged such that nearly all nodes were connected to the cluster by a finite series of steps. In other words, following the topological transition, nearly every author in the scientific field could be connected to any other author by a finite set of co-authorship links: author $A$ wrote a paper with author $B$, who independently wrote a different paper with author $C$, and so forth, until the set of authors became fully connected.

In [17] we hypothesized that such topological transitions signal an important step in a scientific field's development: a distinct, robust scientific field seems to emerge only once there exists some commonly shared set of research questions, concepts, and methods that allow multiple authors to cooperate and collaborate. The topological transition in co-authorship networks, in other words, might provide a signal - available to policymakers and scientists alike - that a new topic has emerged into a full-fledged field of inquiry. Such critical behavior occurs at different times in various fields' evolution, and hence at widely varying sizes of networks. If one relied only upon time-series data such as new authors or publications per year, one would miss the underlying similarities in topology and network structure.

To explore further the dynamics of these topological transitions, and in particular to investigate whether the percolation phenomena betray universality - unfolding in the same way near the transition for a wide variety of fields - we analyze the co-authorship networks using similar techniques to those developed for studying phase transitions in physical systems, such as water freezing to ice. Very powerful quantitative methods have been developed to study the critical dynamics in the vicinity of these transitions [30]. This formalism assumes the thermodynamic limit: the number of nodes is assumed to be both infinite and unchanging. The scientific co-authorship networks of interest to us, however, are always finite, and change in size over time. In addition a general network does not have a well-defined spatial dimensionality, unlike a standard regular lattice. For example, nodes connected in a linear chain form an effectively one-dimensional system. Nodes with more connections 
effectively display higher local dimensionality, which can vary from place to place in the network. These issues lead to a few challenges in the analysis of critical phenomena in evolving networks. To address them we will begin with the usual infinite-size formalism and make modifications to consider finite, time-varying networks, with $1 \ll N<\infty$ and $d N / d t \neq 0$.

Phase transitions are system-wide structural changes in the vicinity of a critical point, such as the freezing point for a liquid-to-ice transition, or the Curie temperature above which a ferromagnet loses its magnetization. In these examples, the critical parameter, $\tau$, depends on the ratio of the system's temperature to its critical value: $\tau=\left[\left(T / T_{c}\right)-1\right]$. In our previous work [17], we found suggestive evidence that fields with $\alpha>1$ might percolate when their average degree, $\langle k\rangle$, crosses some critical field-dependent threshold, $k_{c}$. That is, percolation might occur once the average connectivity of a network, changing in time as in Eq. (2), reaches a critical point. Such behavior would be compatible with previous observations of percolation on random graphs $[31,32,33]$. We therefore define the critical parameter as

$$
\tau \equiv\left[\frac{\langle k\rangle}{k_{c}}-1\right]
$$

and study the dynamics of a given field in the vicinity of $|\tau| \sim 0$. Given the form of Eq. (2), we may rewrite Eq. (3) as

$$
\tau=\left[\left(\frac{N}{N_{c}}\right)^{\alpha_{i}-1}-1\right],
$$

where $N_{c}$ is the size of the network at criticality. Using Eq. (2), we find $k_{c}=E_{i}\left[N_{c}\right]^{\alpha_{i}-1}$. Like a freezing point, the critical degree, $k_{c}$ (and hence $N_{c}$ ), will vary by field. See Table 2.

$\begin{array}{cccccc}\text { field } & E_{i} & \alpha_{i} & N_{c} & k_{c} \text { (estimate) } & k_{c} \text { (measured) } \\ \text { cosmic strings } & 0.28 & 1.21 & 178 & 0.83 & 1.67 \\ \text { inflation } & 0.09 & 1.38 & 121 & 0.56 & 1.59 \\ \text { quantum computing } & 0.38 & 1.22 & 171 & 1.18 & 2.20 \\ \text { scrapie and prions } & 1.53 & 1.12 & 60 & 2.50 & 2.86 \\ \text { string theory } & 0.09 & 1.36 & 79 & 0.43 & 1.24 \\ \text { sustainability science } & 0.15 & 1.27 & 5054 & 1.50 & 2.98 \\ \text { nanotechnology } & 0.07 & 1.36 & 5605 & 1.57 & 3.97\end{array}$

Table 2. Links-per-node scaling coefficients, exponents, and critical network size for the scientific fields in our sample. 
The value of $k_{c}$ at the critical point can be estimated via the scaling relation of edges vs. nodes of Eqs. (1)-(2), which yields the relation $k_{c}=E_{i}\left[N_{c}\right]^{\alpha_{i}-1}$. These values appear in Table 2 in the column, " $k_{c}$ (estimate)." In general, Eq. (1) provides an excellent fit to the data; for example, the fits in Figures $1 \mathrm{C}$ and $1 \mathrm{D}$ each have $R^{2}=0.998$. But the scaling relation of Eq. (1) tends to underestimate the number of links per node for small networks (or, equivalently, at early times in the evolution of large networks), and hence tends to underestimate $\langle k\rangle$ near criticality, at $N=N_{c}$. A direct measure of $k_{c}$ may be made independently of the estimated form, by measuring the average degree, $\langle k\rangle$, at criticality. These values appear in Table 2 in the column, " $k_{c}$ (measured)." Minimizing the discrepancy between these two values of $k_{c}$ remains a matter for future research.

The behavior of the system in the vicinity of $|\tau| \sim 0$ may be characterized by several quantities that scale with $\tau$. The first, $P(\tau)$, is the percolation probability: the likelihood that a randomly-selected node belongs to the largest connected cluster, which may be computed easily as the fraction of nodes in the largest connected cluster. $P(\tau)$ is an order parameter for the system, akin to bulk magnetization for a lattice of spins in a ferromagnet placed in an external magnetic field. The second quantity, $S(\tau)$, is the susceptibility per node, defined as $\sum_{s} s^{2} n_{s} / N$, where $n_{s}$ is the number of clusters that contain $s$ nodes. (The sum extends over all clusters except the largest connected cluster.) $S(\tau)$ thus characterizes the variance of fluctuations per node in cluster size for the system, which is highest at the onset of the formation of a giant graph component, at the critical point. A final quantity of interest is the correlation length, $\xi(\tau)$, which characterizes how smooth or homogenous the system is. Effectively, $\xi(\tau)$ measures the size of the largest clusters that are not part of the single largest component [30].

In the infinite-lattice limit, these quantities scale near $|\tau| \sim 0$ as

$$
\begin{aligned}
& P(\tau) \propto|\tau|^{\beta}, \\
& S(\tau) \propto|\tau|^{-\gamma}, \\
& \xi(\tau) \propto|\tau|^{-\nu},
\end{aligned}
$$


in terms of the so-called critical exponents, $\beta, \gamma$, and $\nu$, with $\beta, \gamma, \nu \geq 0$. Remarkably, many physical systems display "universality": though they freeze at different critical temperatures, $T_{c}$, they all freeze in the same way, that is, they share the same values for the set of critical exponents. Put another way, systems that share the same values of $\beta, \gamma$, and $\nu$ fall into the same universality class. The power of these scaling relationships thus becomes clear: systems that might have quite different microscopic properties and dynamics each behave in exactly the same way near a phase transition [30]. The values of the critical exponents depend on the dimensionality of the system, $d$, and obey a "hyperscaling" relation $[30,35]$

$$
d \cdot \nu=\gamma+2 \beta
$$

In the infinite-volume limit, we therefore see that the percolation probability, $P$, should rise from zero near $|\tau| \sim 0$ while the susceptibility, $S$, and correlation length, $\xi$, should diverge. In a finite system, however, the correlation length can only grow as large as the system itself: $\xi \leq N$. Likewise, in a finite system clusters can only grow so large, so $S$ will reach some maximum but finite value near $|\tau| \sim 0$, rather than diverging to infinity; while $P$ will remain at some small but nonzero value near $|\tau| \sim 0$. See Figure 2 .

In order to account for the finite size of our systems, $1 \ll N<\infty$, we make use of the usual scaling hypothesis (see, e.g., Section 2.3 of [35]). At any given time, the only length scales are the smallest scale in the system (an individual author), the total number of authors, $N$, and the correlation length, $\xi$. From Eq. (5), we see that $\tau \propto \xi^{-1 / \nu}$. Since we assume that the only dimensionful quantity of relevance to the dynamics is $\tau$, we scale the quantities $P$ and $S$ as

$$
\begin{aligned}
& P(\tau)=[\xi(\tau)]^{-\beta / \nu} P_{0}(N / \xi), \\
& S(\tau)=[\xi(\tau)]^{\gamma / \nu} S_{0}(N / \xi),
\end{aligned}
$$

where the quantities $P_{0}$ and $S_{0}$ depend only on the dimensionless ratio, $N / \xi$. These functions obey the following limits:

$$
\begin{array}{ccc}
\xi \ll N: & P_{0} \rightarrow C_{0}, & S_{0} \rightarrow D_{0}, \\
\xi \rightarrow N: & P_{0} \rightarrow C_{0}\left(\frac{N}{\xi}\right)^{-\beta / \nu}, & S_{0} \rightarrow D_{0}\left(\frac{N}{\xi}\right)^{\gamma / \nu},
\end{array}
$$



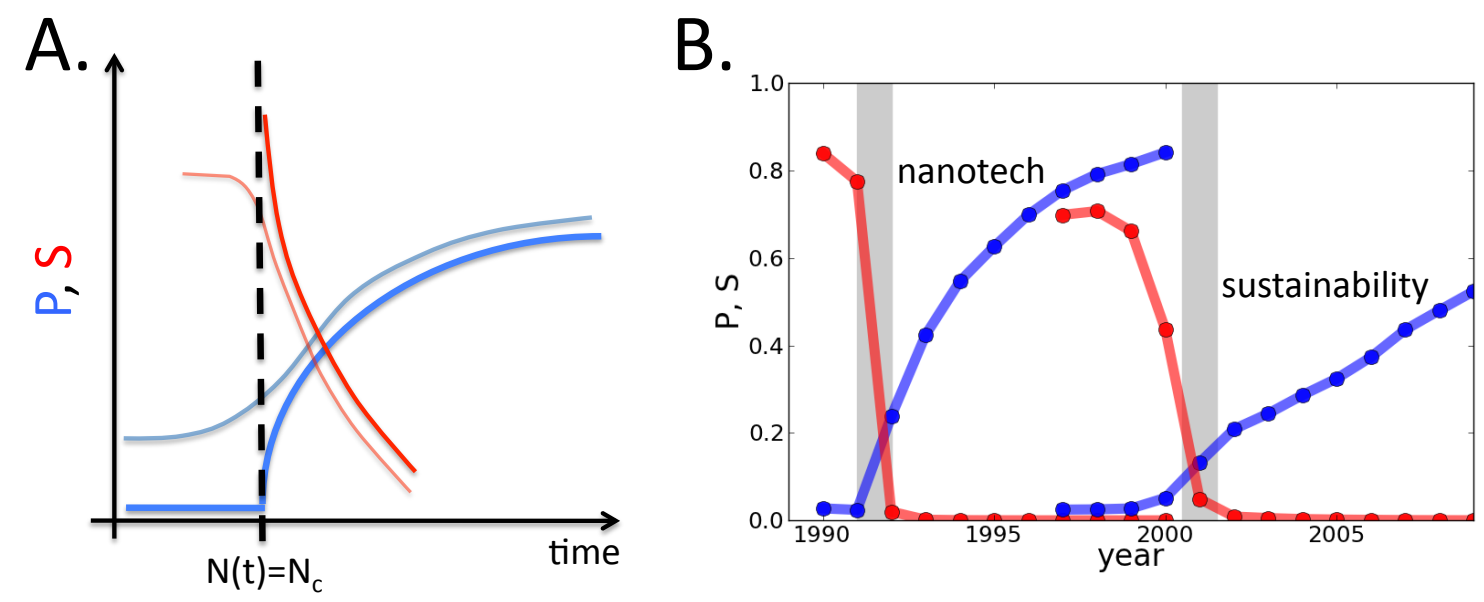

Figure 2: Signatures of the topological transition at the advent of the formation of scientific fields. A. Schematic representation of the behavior of the percolation cumulants $P$ (blue) and $S$ (red). In the idealized infinite-size limit, as a field forms and densifies, it undergoes a topological transition such that $P$ grows away from zero while $S$ quickly decays from a large value; in this limit, $P$ has a derivative discontinuity at the critical point, while $S$ has a divergence at the critical point. The behavior of these quantities for real fields are finite-size approximations (pale lines) to their infinite-size extrapolations (bright lines). B. The corresponding critical behavior for two large fields in our sample, nanotechnology and sustainability science.

where $C_{0}$ and $D_{0}$ are constants. Thus, it is clear that near the critical point, as the correlation length diverges to be comparable to the size of the entire system $(\xi \rightarrow N)$, we obtain

$$
\begin{aligned}
& P \rightarrow C_{0} N_{c}^{-\beta / \nu}, \\
& S \rightarrow D_{0} N_{c}^{\gamma / \nu},
\end{aligned}
$$

where $N_{c}$ is the size of the network at criticality.

We may make a further rescaling in order to extract the dependence on the system size, $N$, by writing

$$
\begin{aligned}
& P_{0}(N / \xi)=\left(\frac{N}{\xi}\right)^{-\beta / \nu} \tilde{P}(N / \xi), \\
& S_{0}(N / \xi)=\left(\frac{N}{\xi}\right)^{\gamma / \nu} \tilde{S}(N / \xi) .
\end{aligned}
$$

Eq. (8) requires that the new functions obey

$$
\begin{array}{ccc}
\xi \ll N: & \tilde{P} \rightarrow C_{0}\left(\frac{N}{\xi}\right)^{\beta / \nu}, & \tilde{S} \rightarrow D_{0}\left(\frac{N}{\xi}\right)^{-\gamma / \nu} \\
\xi \rightarrow N: & \tilde{P} \rightarrow C_{0}, & \tilde{S} \rightarrow D_{0},
\end{array}
$$


while Eqs. (7) and (10) require

$$
\begin{aligned}
& P(\tau)=N^{-\beta / \nu} \tilde{P}(N / \xi), \\
& S(\tau)=N^{\gamma / \nu} \tilde{S}(N / \xi) .
\end{aligned}
$$

In order to retain the appropriate scaling with $\tau$, as in Eq. (5), we therefore see that the $\operatorname{argument}$ of $\tilde{P}$ and $\tilde{S}$ must be

$$
\left(\frac{N}{\xi}\right)^{1 / \nu}=N^{1 / \nu} \tau .
$$

Combining Eqs. (11), (12), and (13), we therefore find

$$
\begin{aligned}
& P(\tau)=C_{0} N^{-\beta / \nu} f\left(N^{1 / \nu} \tau\right), \\
& S(\tau)=D_{0} N^{\gamma / \nu} g\left(N^{1 / \nu} \tau\right) .
\end{aligned}
$$

Plotting $P /\left[C_{0} N^{-\beta / \nu}\right]$ versus $N^{1 / \nu} \tau$ should then yield a single curve, $f$, on which all members of the same universality class fall. Likewise, plotting $S /\left[D_{0} N^{\gamma / \nu}\right]$ versus $N^{1 / \nu} \tau$ should yield a single curve, $g$, on which all members fall.

Our empirical results are consistent with the hypothesis of universality. First consider Figure 3, which shows the scaling of $P$ and $S$ for each scientific field at criticality, $N=N_{c}$. Note how closely all seven fields in our sample obey the predicted scaling with $N_{c}$ as given by Eq. (9). All seven fields fall upon a single line for $P$ and $S$, with exponents given by $\beta / \nu=0.36$ (95\% confidence interval $\left.[0.27,0.44], R^{2}=0.93\right)$ and $\gamma / \nu=0.31$ (95\% confidence interval $\left.[0.19,0.42], R^{2}=0.85\right)$. The constant coefficients take the values $C_{0}=0.52$ and $D_{0}=0.66$.

Using these estimates leads to predictions for the exponents $\beta$ and $\gamma$, given the value of $\nu$. In practice we investigate the dependence of $P$ and $S$ on the critical parameter, $\tau$, directly as we vary $\nu$, as in Figures 4 and 5. These, too, scale as expected in the light of Eq. (14), and provide a consistency check that there is a single set of exponents that describe (at least approximately) all fields at the transition. Figure 6 shows the allowed region for these parameters as the intersection of the uncertainty ranges in the fits of Figs. 3-5, plus the dimensional limits arising from the hyperscaling relation of Eq. (6). We see that the 
allowed region corresponds to an effective dimensionality $d \gtrsim 1$. The best parameters are obtained at

$$
\nu=1.21 \pm 0.01, \beta=0.45 \pm 0.04, \gamma=0.35 \pm 0.03, \rightarrow d=1.04 \pm 0.07
$$

where errors are computed at $95 \%$ confidence. At $d=1$ we obtain the best parameter estimates

$$
\nu=1.31 \pm 0.01, \beta=0.47 \pm 0.03, \gamma=0.36 \pm 0.03
$$

These estimates still suffer from some uncertainties, as most fields show only limited and noisy scaling with $\tau$ in the critical region. Nevertheless, the estimates of a low dimensionality

for the transition, with $d \gtrsim 1$, suggest that the networks are structured as strings of dense cliques, each clique weakly linked to the next; that is, as blobs on a line. Within each clique one finds high local degree, $k \gg 1$, with loose connections from a few nodes to the next neighboring clique. Large-scale connectivity — of the sort that allows the entire network to percolate into a single connected component — thus behaves essentially as a linear (onedimensional) chain of collaboration. We discuss these aspects of the transition and the value of the exponents below in greater detail.

\subsection{Network Structure and Micro-Dynamics}

All seven of the scientific fields in our sample — ranging over nearly four orders of magnitude in size - betray self-similar behavior in the vicinity of their critical points. That is, the bulk properties of their topological transition are independent of the micro-level dynamics by which their networks evolve. Nonetheless, simple scaling relations like Eq. (1), showing the densification over time as the networks grow, suggest that we may be able to model the micro-level structure and dynamics of these networks as well.

The goal of this subsection is to find some form for the underlying degree distribution, $p_{k}$, which could be consistent with the bulk properties near criticality. Here $p_{k}$ gives the probability that a randomly chosen node has degree $k$. The degree distribution, $p_{k}$, thus 

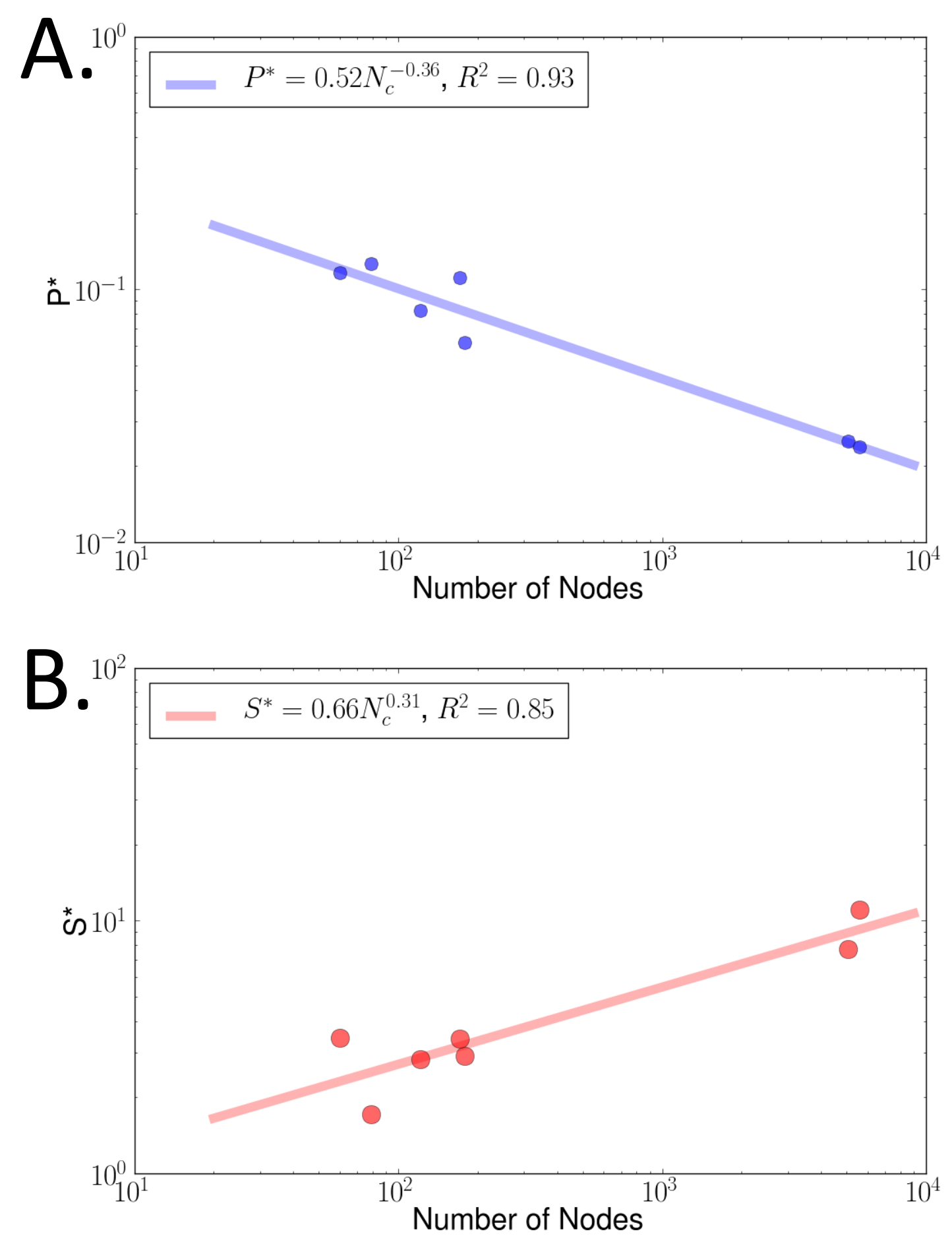

Figure 3: The finite-size scaling of $P$ and $S$ at criticality for seven fields with different sizes. A. The percolation probability, $P$, decays to zero as a scale-invariant function of $N$ with exponent $\beta / \nu=0.36$ (95\% confidence interval $[0.27,0.44]$ ) . B. The susceptibility, $S$, diverges to infinity with increasing $N$, with an exponent $\gamma / \nu=0.31$ (95\% confidence interval $[0.19,0.42])$. The behavior of both of these quantities suggests the existence of an infinite-size idealized topological transition to which all fields in our sample are finite-sized realizations. 


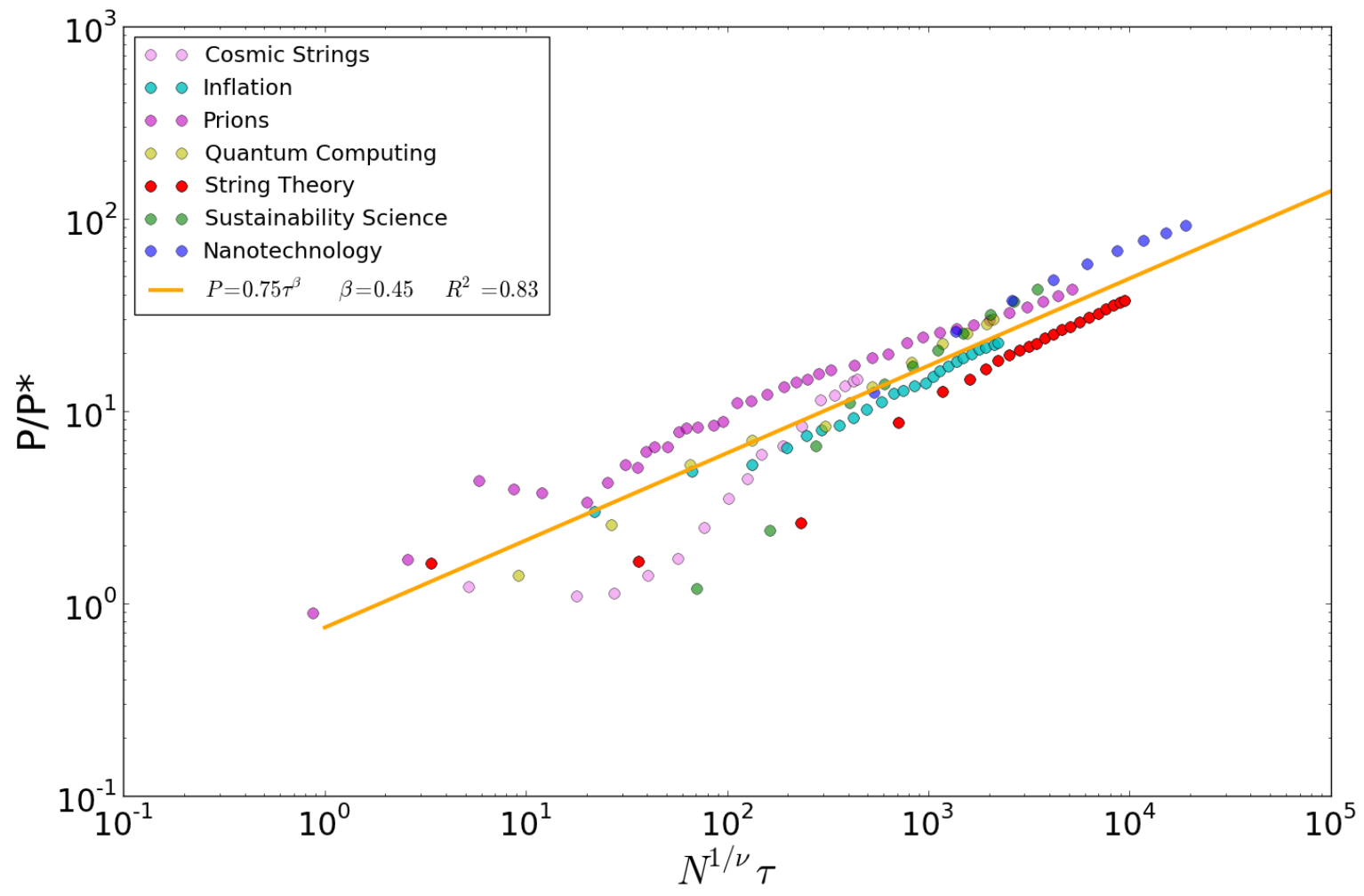

Figure 4: The behavior of the percolation probability, $P$, with $\tau$ for our set of scientific fields. Plotted here is $P / P^{*}$ versus $N^{1 / \nu} \tau$, where $P^{*} \equiv C_{0} N^{-\beta / \nu}$. The yellow line shows the expected scaling for the particular value of the critical exponents, $\beta=0.45, \nu=1.21$. 


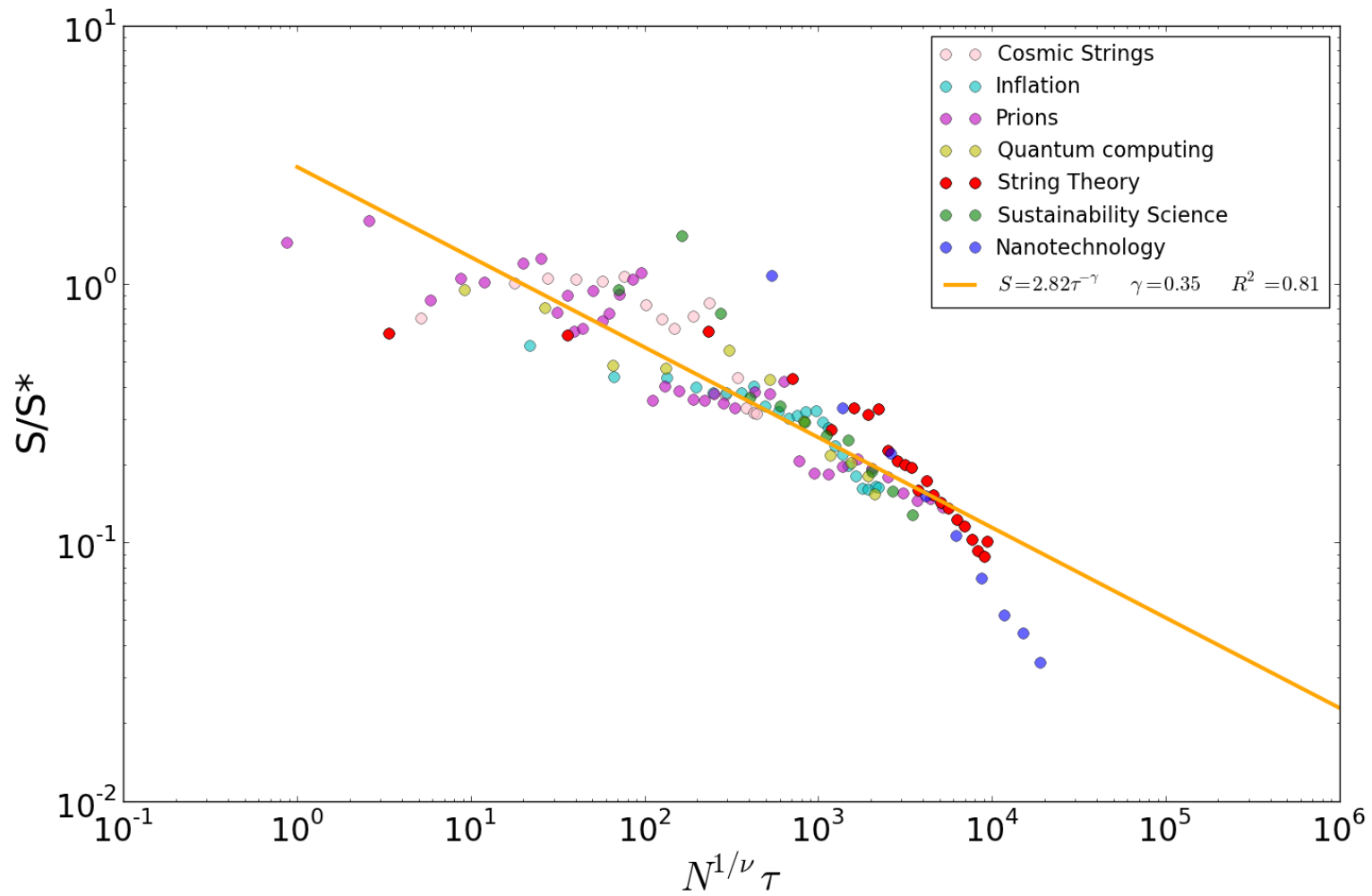

Figure 5: The behavior of the susceptibility, $S$, with $\tau$ for our set of scientific fields. Plotted here is $S / S^{*}$ versus $N^{1 / \nu} \tau$, where $S^{*} \equiv D_{0} N^{-\gamma / \nu}$. The yellow line shows the expected scaling for the particular value of the critical exponent, $\gamma=0.35, \nu=1.21$. 


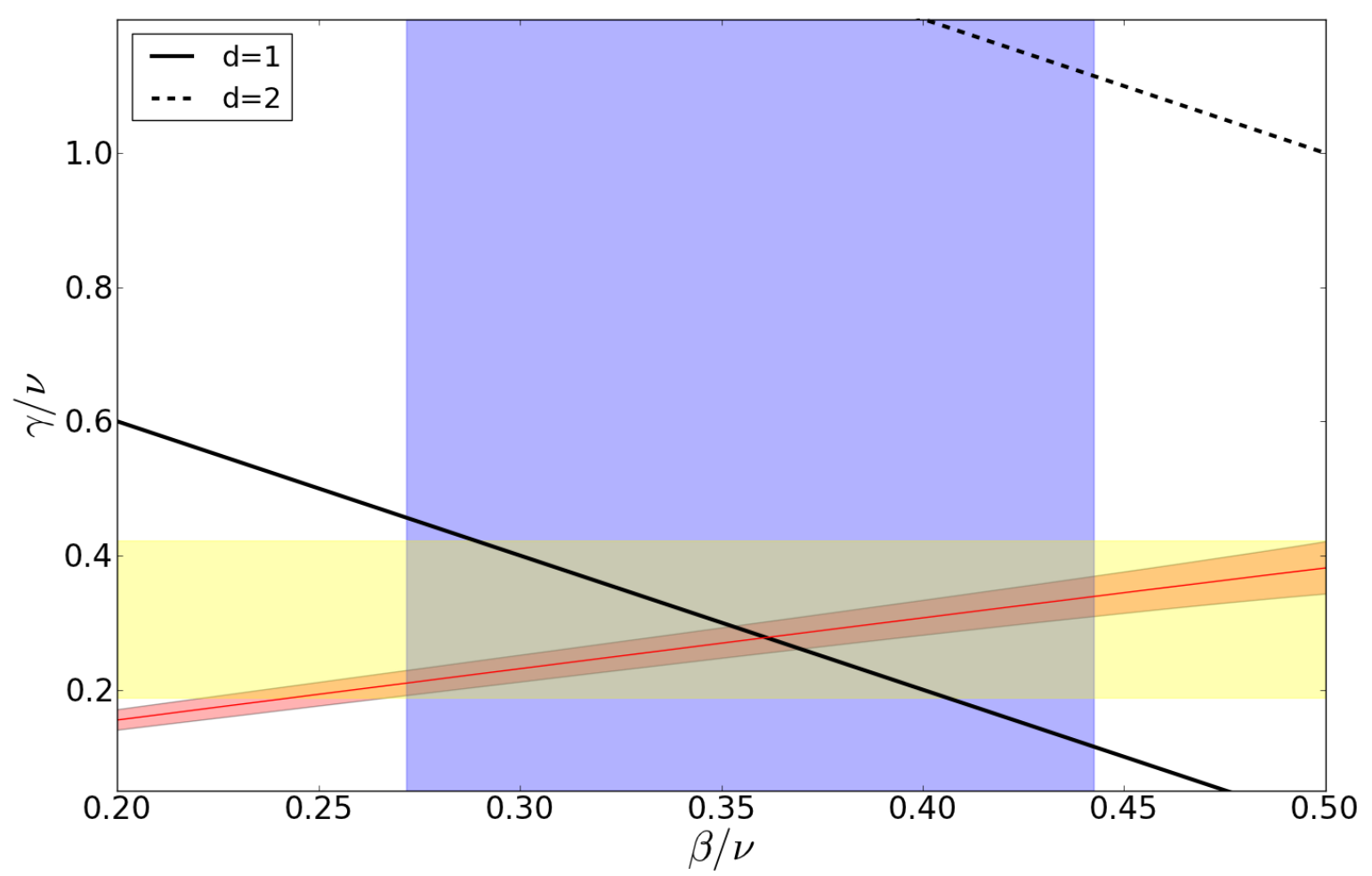

Figure 6: The allowed parameter space of exponents resulting from the finite-size scaling at criticality of $P$ and $S$ (blue and yellow regions, respectively) and direct estimate of their dependence on $\tau$ (red region), see Figs 4-5. The red line shows the trajectory of the two ratios $\beta / \nu$ and $\gamma / \nu$ as a function of $\nu$. Solid and dashed lines show dimensionality constraints on the exponent ratios resulting from the hyperscaling relation $d=2 \beta / \nu+\gamma / \nu$. The allowed range for the ratios at $95 \%$ confidence corresponds to the intersection of the yellow, blue and red regions, above the solid black line. In this region $\nu$ ranges from 1.31 for which $\beta=0.47$ and $\gamma=0.36$ (with $d=1$ ), and $\nu=0.75$, with $\beta=0.33$ and $\gamma=0.25$. The best fits are obtained in the vicinity of $\nu \sim 1.21$, with $\beta=0.45$ and $\gamma=0.35, d=1.04$. These results suggest that a common description of all collaboration networks corresponds to a non-standard percolation phenomenon dominated by chains of collaboration at criticality, corresponding to an effective dimension $d \gtrsim 1$. 
plays an analogous role to the partition function in statistical mechanics. As in statistical mechanics, $p_{k}$ should offer a bridge between the micro-level dynamics of the system and the bulk (quasi-thermodynamic) properties near the phase transition.

Many networks across the physical, biological, and social domains feature degree distributions that are close to power-law form, $p_{k} \sim k^{-s}$, at least over some wide range of degree, $k$. That is, they contain many nodes with a small number of links, and few nodes with many links. As noted above, real-world networks of interest grow large but remain finite, with $1 \ll N<\infty$. In such finite networks, the pure power-law distribution is often truncated: $p_{k} \sim k^{-s} f(k)$, where $f(k)$ is a rapidly-falling function of $k$. (For reviews, see $[31,32,33]$.) Because are interested in late-time and large-size effects, we will make use of the continuum approximation throughout this subsection: $\sum_{k} p_{k} \rightarrow \int d k p(k)$.

Previous studies of scientific collaboration networks have modeled the degree distributions by a soft (exponential) cutoff of the form $f(k)=\exp [-k / \kappa][10]$. We therefore consider a degree distribution of the form

$$
p_{i}(k)=\left\{\begin{array}{cc}
0 & \text { for } k=0 \\
\mathcal{C}_{i} k^{-s_{i}} e^{-k / \kappa_{i}} & \text { for } k \geq 1
\end{array}\right.
$$

Given the cutoff parameter, $\kappa_{i}$, we may integrate up to $k \rightarrow \infty$ in the continuum limit. The constant $\mathcal{C}_{i}$ is determined from normalization, and therefore the degree distribution for each field, $p_{i}(k)$, depends on only two free parameters: the scaling exponent, $s_{i}$, and the cutoff parameter, $\kappa_{i}$. The exponent, $s_{i}$, determines how likely it is to find a well-connected author with $k$ links, while $\kappa_{i}$ incorporates finite-size effects; hence we will assume that $\kappa_{i}$ scales in some way with the network size, $N$. Just as each field grew and densified according to the same basic scaling relation of Eq. (1) but with different values for $E_{i}$ and $\alpha_{i}$, we expect that each field has an underlying degree distribution of the form in Eq. (17), with its own values for $s_{i}$ and $\kappa_{i}$.

First we determine the normalization constant, $\mathcal{C}_{i}$. The incomplete Gamma function, 
$\Gamma(a, z)$, is defined by the integral $[36]$

$$
\Gamma(a, z)=\int_{z}^{\infty} d t t^{a-1} e^{-t}
$$

and thus we find

$$
1=\int_{0}^{\infty} d k p_{i}(k)=\int_{1}^{\infty} d k \mathcal{C}_{i} k^{-s_{i}} e^{-k / \kappa_{i}}=\kappa_{i}^{1-s_{i}} \Gamma\left(1-s_{i}, 1 / \kappa_{i}\right),
$$

or

$$
\mathcal{C}_{i}=\frac{\kappa_{i}^{s_{i}-1}}{\Gamma\left(1-s_{i}, 1 / \kappa_{i}\right)} .
$$

The average degree then takes the form (dropping the field-index $i$ for convenience)

$$
\langle k\rangle=\int_{0}^{\infty} d k k p(k)=\int_{1}^{\infty} d k \mathcal{C} k^{1-s} e^{-k / \kappa}=\kappa \frac{\Gamma(2-s, 1 / \kappa)}{\Gamma(1-s, 1 / \kappa)} .
$$

Using the general properties of the $\Gamma(a, z)$ functions [36], we find the leading behavior of Eq. (21) in the $\kappa \gg 1$ limit to be

$$
\langle k\rangle=\left\{\begin{array}{cc}
(s-1) \Gamma(2-s) \kappa^{2-s} & \text { for } 1<s<2 \\
(s-1)(s-2)^{-1} \kappa^{0} & \text { for } s>2
\end{array}\right.
$$

The apparent divergence in $\langle k\rangle$ at $s=2$ is an artifact. Expanding $\Gamma(0,1 / \kappa)$ in the limit $\kappa \gg 1$, we have [36]

$$
\Gamma(0,1 / \kappa)=\ln \kappa-\gamma_{E}+\kappa^{-1}+\mathcal{O}\left(\kappa^{-2}\right)
$$

where $\gamma_{E}=0.5772 \ldots$ is the Euler-Mascheroni constant. This yields

$$
\langle k\rangle_{s=2}=\mathcal{C} \Gamma(0,1 / \kappa)=\ln \kappa+\mathcal{O}\left(\kappa^{0}\right) .
$$

Thus the average degree will only scale as some power of network size, $N$, for exponents in the range $1<s_{i}<2$.

We now assume some relation between the exponential cutoff parameter, $\kappa_{i}$, and network size, $N$. The cutoff effectively measures the largest degree found in the network, $\kappa_{i} \sim k_{\max }$. Clearly no node can have more links than there are other nodes in the network, so $\kappa_{i}$ must be bounded from above by $\kappa_{i}<(N-1) \sim N$. Moreover, at any finite time authors in a network 
might not yet have had a chance to form links with all the other potential co-authors. Thus we expect the cutoff parameter to scale with network size as

$$
\kappa_{i}=N^{b_{i}} \text {, with } 0<b_{i}<1
$$

Equating the two expressions for $\langle k\rangle$ in Eq. (2) and (22), we find, for $1<s_{i}<2$,

$$
E_{i}=\left(s_{i}-1\right) \Gamma\left(2-s_{i}\right)
$$

and

$$
\alpha_{i}=1+b_{i}\left(2-s_{i}\right)
$$

From Eq. (27), we see that networks will only densify, with $\alpha_{i}>1$, if their underlying degree distribution has a scaling exponent $s_{i}<2$. If $p(k)$ falls off more rapidly than $k^{-2}$ then too few nodes in the network will be well enough connected to allow the overall network to densify and percolate.

Using our empirical values for $E_{i}$ and $\alpha_{i}$ in Table 2 together with Eqs. (26) and (27), we find

$\begin{array}{ccc}\text { field } & s_{i} & b_{i} \\ \text { cosmic strings } & 1.23 & 0.27 \\ \text { inflation } & 1.09 & 0.42 \\ \text { quantum computing } & 1.30 & 0.31 \\ \text { scrapie and prions } & 1.63 & 0.33 \\ \text { string theory } & 1.09 & 0.39 \\ \text { sustainability science } & 1.14 & 0.31 \\ \text { nanotechnology } & 1.07 & 0.39\end{array}$

Table 3. Degree distribution scaling exponents and cutoff parameter scalings for the scientific fields in our sample.

The field with the largest scaling exponent — and hence the steepest fall-off in likelihood for multiple co-author links per author — is the biomedical field of scrapie and prions, with $s_{i}=1.63$. Several areas of theoretical physics, on the other hand, such as inflation and string theory, have smaller scaling exponents (more frequent large-degree nodes) with $s_{i}=1.09$. Likewise, in a previous study [10], Newman found that the network of authors who had 
published biomedical articles registered in Medline (about 1.4 million authors ca. 2000) were relatively loosely connected, with a large scaling exponent, $s_{i}=2.5$, whereas most physics fields had $s_{i} \simeq 1.1-1.3$. He also found cutoff parameters that scaled in the range $0.25 \leq b_{i} \leq 0.65$

Perhaps the biggest surprise of our analysis is that our largest and most heterogeneous field, nanotechnology, appears to have the smallest scaling exponent, $s_{i}=1.07$. One might have expected instead that an interdisciplinary field as large and diverse as nanotechnology would behave more like the network of all authors in Medline, rather than like a small and relatively homogenous field like cosmological inflation or string theory. This curious finding deserves further study.

\subsection{Finer Substructure?}

The simple ansatz for the underlying degree distribution, $p(k)$ as in Eq. (17), can reproduce the bulk properties we had found for our various fields near their critical point. But $p(k)$ can also be measured directly at any given moment in the evolution of a network, by plotting the frequency of a given number of links within a network at a given time. How well does the form of Eq. (17) match such snapshot measurements?

Figure 7 shows the degree distribution near $N=N_{c}$ for several fields. The orange line shows the scaling $p(k)=k^{-2}$ for reference. Though most cases clearly fall off as a quasipower-law with increasing $k$, they are much more flat at small $k$ than the form of Eq. (17) would suggest, and they fall off more steeply than $k^{-s}$ with $1<s_{i}<2$.

The discrepancy between the assumed form of $p(k)$ and the data in Figure 7 might be accommodated by modifying our assumption about the micro-dynamics by which the networks grow. The degree distribution of Eq. (17) incorporates the assumption that each new author joins the network as an individual. Yet the value of the bulk scaling exponents, $\beta / \nu=0.36$, which we found above, might suggest the presence of significant "clique" substructure. That is, our scientific fields might not grow as pure random graphs, with each new node added 


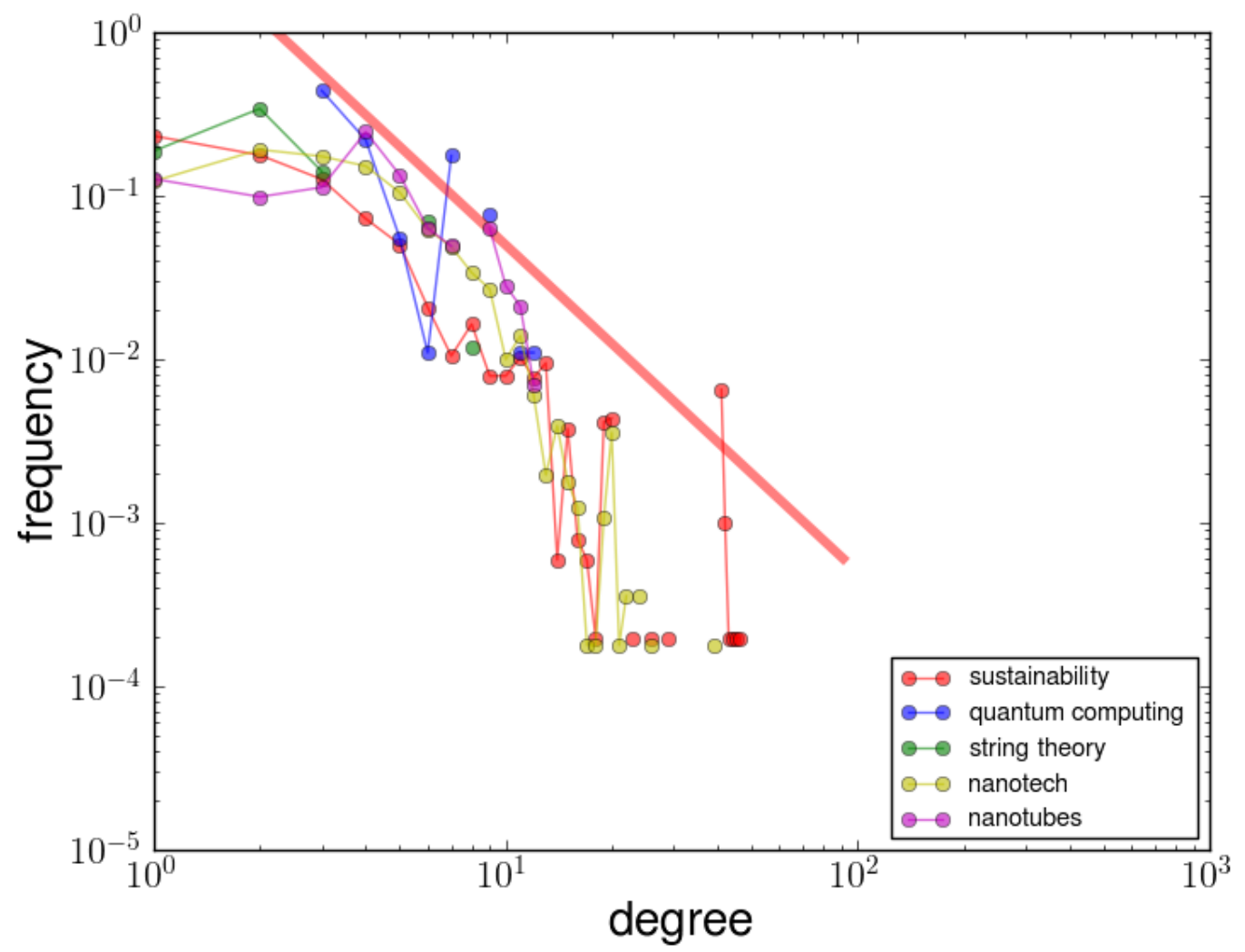

Figure 7: The degree distribution, $p(k)$, versus $k$ for several fields, as measured in the vicinity of each field's critical point, $N=N_{c}$. The orange line shows the pure power-law distribution, $p(k) \sim k^{-2}$. Unlike the plots in Figures 3-5, there is no reason to expect each field's degree distribution to line up on a single curve, since the free parameters within $p(k)$ - namely, $s_{i}$ and $\kappa_{i}$ - vary by field. Nevertheless, we expect $p(k)$ for each field to fall roughly as a power-law with increasing degree, up to some finite-size cutoff. 
autonomously of all others. Rather, new nodes might join the network in pre-formed clusters or "cliques" of size $r$. As found in [37], the order parameter in such a case should scale with network size as

$$
\Psi= \begin{cases}N_{c}^{-r / 6} & \text { for } r \leq 3 \\ N_{c}^{1-r / 2} & \text { for } r \geq 3 .\end{cases}
$$

Our finding in Eq. (9) for the scaling of the order parameter, $P \sim N_{c}^{-\beta / \nu}$, with $\beta / \nu=0.36$, is close to the predicted scaling of Eq. (28) for $r=2$, that is, for networks in which each new author joins the network as part of a small clique of two co-authors. We might therefore modify the degree distribution of Eq. (17) to take the form

$$
p(k)=\mathcal{C}_{i}(k+r)^{-s_{i}} \exp \left[-(k+r) / \kappa_{i}\right]
$$

Proceeding as in the previous subsection, we find

$$
\mathcal{C}_{i}=\frac{\kappa_{i}^{s_{i}-1}}{\Gamma\left(1-s_{i}, \Lambda_{i}\right)},
$$

where

$$
\Lambda_{i} \equiv \frac{(1+r)}{\kappa_{i}} \ll 1
$$

and thus, for $1<s_{i}<2$,

$$
\langle k\rangle=\left(s_{i}-1\right) \Gamma\left(2-s_{i}\right)(1+r)^{s_{i}-1} \kappa_{i}^{2-s_{i}}+\mathcal{O}\left(\kappa_{i}^{0}\right) .
$$

Again assuming that the cutoff parameter scales with network size as $\kappa_{i}=N^{b_{i}}$, and comparing with Eq. (2), we thus find

$$
E_{i}=\left(s_{i}-1\right) \Gamma\left(2-s_{i}\right)(1+r)^{s_{i}-1}
$$

and

$$
\alpha_{i}=1+b_{i}\left(2-s_{i}\right)
$$

The sum-rule among the exponents in Eq. (34) is unchanged from our previous finding in Eq. (27), but the relation among coefficients in Eq. (33) has picked up the extra factor of $(1+r)^{s_{i}-1}$ compared to the corresponding expression in Eq. (26). For $r=2$ and $s_{i} \sim 1.1-1.6$, 
this extra factor amounts to a multiplicative constant of $1.12-1.93$. The overall effect of the $r$-clique modification is thus to flatten out the degree distribution at small $k$, to better match the empirical findings in Figure 7, while retaining the large- $N$ behavior of $\langle k\rangle$, and hence of the percolation behavior described in previous subsections.

Whether an underlying degree distribution of the form in Eq. (29) or some other form will best capture the underlying dynamics remains a topic for further study.

\section{Discussion}

Scientific fields are self-organizing collections of people, their knowledge and interactions and the physical products of their research evolving over time, all aimed at solving specific problems. Mature fields of science are unified by subject matter, research techniques, conferences and journals. However, the most interesting stage of field formation is also the most uncertain and deals with the moment at which the advent of new concepts and techniques produces new communities of practice that eventually become mature fields. It is just before and at this point that policy interventions can be expected to be most effective.

The present study deals with a central question in epistemology and the emerging science of science, namely whether there exist general processes common across disciplines, time, and scale that characterize the formation of new fields of science. If such common processes do exist, then quantification of such processes would provide a unified framework to understand and predict the evolution and structure of every scientific field, with important implications to science as well as policy. This would mean, for example, that there should be general objectives of science policy that will benefit any field of research, regardless of its size or disciplinary focus.

There have been a number of tantalizing pieces of evidence to suggest that such a unified description does exist, and that it relates to a (geometric) critical phenomenon, crossed at a finite rate in time and at a finite (and often small) scale in terms of network size. Here we have developed additional evidence in support of this picture and proposed a quantitative 
framework in which to generate features of dynamics and network structure that can be accounted for and parameterized in terms of a few universal parameters (critical exponents). While more evidence is still necessary, the present study is strongly suggestive that a universal critical structural change in interactions between scientists marks the advent of a new field.

To establish the values of critical exponents and the extent to which these are universal, more examples of fields, across an even greater range of scales and diversity of research areas, will be necessary. We found that the inclusion of larger fields - nanotechnology and sustainability science - was actually crucial to enable us to glimpse details of behavior in the critical region, especially to test the scaling assumption behind Eq. (9) and demonstrated in Figure 3. The critical dynamics are sensitive to $N$, and hence testing the expected relations across a wide range of $N$ is essential.

Many of the smaller fields that we have examined in this study show scaling only over a very small window of their dynamics. This is familiar from studies of critical phenomena in small systems, where the most spectacular signatures of criticality are washed out. We have begun to explore other possible forms for the critical parameter, $\tau$, which might prove more sensitive to the critical dynamics of smaller fields. For example, rather than parameterizing $\tau$ in terms of the average degree, $\langle k\rangle$, as in Eq. (3), we could incorporate fluctuations around the average degree, $\left\langle k^{2}\right\rangle$. The natural combination to consider would take the form $\tau=(T-1)$, where $T=\left\langle k^{2}\right\rangle /(2\langle k\rangle)$ (see $\left.[33,38]\right)$. Whether this new ansatz for $\tau$ will prove any better empirically at capturing sensitive features of smaller networks' growth remains a topic for further study.

If all fields show differences of critical exponents under a more extensive set of examples, then this raises the question of whether each field undergoes the same critical phenomenon or if instead there are universality classes that characterize different sets of fields, each with their own values for $\beta, \gamma$, and $\nu$. On the other hand, if all fields can be associated with the same set of critical exponents - as it strongly appears that they can, given the present analysis - then the next challenge to tackle will be to develop and test more accurate 
models of the underlying, micro-level dynamics that give rise to the observed behavior of universal structural change. The results of our empirical analysis developed here suggest a low effective dimensional model where, around the time of field formation, distant authors are connected primarily in terms of linear $(d=1)$ chains of collaboration. This does not preclude that locally most publications are co-authored by several people and that many authors enjoy large numbers of collaborators, leading to dense collaboration clusters. However, these cliques remain for a while disconnected as isolated groups, and field formation occurs when their interconnectivity is just high enough to lead to a giant graph component, close to effectively a one-dimensional set of chains over the longest distances in the network. Because one-dimensional critical phenomena are simpler and often analytically tractable, these findings suggest the existence of a simple model that can account for the observed critical dynamics of fields of any size. Such a model will necessarily be different from standard

percolation in order to account for the observed exponents. For example, the presence of clique substructure as new authors enter a given field (as discussed in Section 2.4) would produce a departure from pure random graph theory, and might well account for the values of critical exponents found here.

The existence of a general theory and detailed model that describes field formation across disciplines, time, and population size would provide a new comprehensive, quantitative, and predictive framework with which to understand the social and conceptual dynamics involved in the self-organized creation of scientific communities of practice. Such a framework would hold great promise for guiding science policy.

\section{Acknowledgments}

This work was partially supported by the DOE Office of Scientific and Technical Information (OSTI). We also gratefully acknowledge assistance from Jasleen Kaur, Shreeharsh Kelkar, and Andrei Mogoutov for help in constructing and parsing the database of publications on nanotechnology. 


\section{References}

[1] W. Goffman and V. A. Newill, "Generalization of epidemic theory: An application to the transmission of ideas," Nature 204 (1964): 225-228.

[2] W. Goffman, "Mathematical approach to the spread of scientific ideas: The history of mast cell research," Nature 212 (1966): 449-452.

[3] W. Goffman and G. Harmon, "Mathematical approach to the prediction of scientific discovery," Nature 229 (1971): 103-104.

[4] E. Garfield, "The epidemiology of knowledge and the spread of scientific information," Current Contents 35 (1980): 5-10.

[5] A. N. Tabah, "Literature dynamics: Studies of growth, diffusion, and epidemics," $A n$ nual Review of Information Science and Technology (ASIS) 34 (1999): 249-286.

[6] L. M. Bettencourt, A. Cintron-Arias, D. I. Kaiser, and C. Castillo-Chavez, "The power of a good idea: Quantitative modeling of the spread of ideas from epidemiological models," Physica A 364 (2006): 513-536.

[7] L. M. Bettencourt, D. I. Kaiser, J. Kaur, C. Castillo-Chávez, and D. E. Wojick, "Population modeling of the emergence and development of scientific fields," Scientometrics 75 (2008): 495-518.

[8] D. J. de Solla Price, "Networks of scientific papers," Science 149 (1965): 510-515.

[9] S. Redner, "Citation statistics from 110 years of Physical Review," Physics Today 58 (6) (2005): 49-54.

[10] M. E. J. Newman, "The structure of scientific collaboration networks," Proceedings of the National Academy of Sciences USA 98 (2001): 404-409 [arXiv:cond-mat/0007214]. 
[11] M. E. J. Newman, "Scientific collaboration networks, I. Network construction and fundamental results," Physical Review E 64 (2001): 016131.

[12] M. E. J. Newman, "Scientific collaboration networks, II. Shortest paths, weighted networks, and centrality," Physical Review E 64 (2001): 016132.

[13] A. L. Barabasi, H. Jeong, Z. Neda, E. Ravasz, A. Schubert, and T. Vicsek, "Evolution of the social network of scientific collaborations," Physica A 311 (2002): 590-614.

[14] M. E. J. Newman, "Who is the best connected scientist? A study of scientific coauthorship networks," Lecture Notes in Physics 650 (2004): 337-370.

[15] J. J. Ramasco, S. N. Dorogovtsev, and R. Pastor-Satorras, "Self-organization of collaboration networks," Physical Review E 70 (2004): 036106.

[16] K. Börner, L. Dall'Asta, W. Ke, and A. Vespignani, "Studying the emerging global brain: Analyzing and visualizing the impact of co-authorship teams," Complexity 10 (2005): 57-67.

[17] L. M. Bettencourt, D. I. Kaiser, and J. Kaur, "Scientific discovery and topological transitions in collaboration networks," Journal of Informetrics 3 (2009): 210-221.

[18] R. M. Shiffrin and K. Börner, "Mapping knowledge domains," Proceedings of the National Academy of Sciences USA 98 (2001): 5183-5185.

[19] K. W. Boyack, R. Klavans, and K. Börner, "Mapping the backbone of science," Scientometrics 64 (2005): 351-374.

[20] J. Bollen, H. van de Sompel, H. Haberg, L. Bettencourt, R. Chute et al., "Clickstream data yields high-resolution maps of science," PLoS One 4 (3) (2009): e4803, doi:10.1371/journal.pone.0004803.

[21] K. Börner, Atlas of Science: Visualizing What We Know (Cambridge: MIT Press, 2010). 
[22] D. Chavalarias and J. Cointet, "Science mapping with asymmetric co-occurrence analysis: Methodology and case study," in Proceedings of the European Conference on Complex Systems, Dresden (2007), pp. 1-5.

[23] D. Chavalarias and J. Cointet, "Bottom-up scientific field detection for dynamical and hierarchical science mapping: Methodology and case study," Scientometrics 75 (2008): $37-50$.

[24] T. S. Kuhn, The Structure of Scientific Revolutions (Chicago: University of Chicago Press, 1962, 1970).

[25] D. J. de Solla Price, Little Science, Big Science (New York: Columbia University Press, 1963).

[26] J. E. Hirsch, "An index to quantify an individual's scientific research output," Proceedings of the National Academy of Sciences USA 102 (2005): 16569-16572.

[27] A. Mogoutov and B. Kahane, "Data search strategy for science and technology emergence: A scalable and evolutionary query for nanotechnology tracking," Research Policy 36 (2007): 893-903.

[28] L. M. Bettencourt, and J. Kaur, "Mapping the evolution and structure of sustainability science", in review.

[29] J. Leskovec, J. Kleinberg, and C. Faloutsos, "Graphs over time: Densification laws, shrinking diameters, and possible explanations," Proceedings of the Eleventh ACM SIGKDD International Conference on Knowledge Discovery in Data Mining (New York: ACM, 2005), 177-187.

[30] J. Zinn-Justin, Quantum Field Theory and Critical Phenomena (New York: Oxford University Press, 1989). 
[31] R. Albert and A.-L. Barabási, "Statisical mechanics of complex networks," Reviews of Modern Physics 74 (2002): 47-97 [arXiv:cond-mat/0106096].

[32] S. N. Dorogovtsev and J. F. F. Mendes, "Evolution of networks," Advances in Physics 51 (2002): 1079-1187 [arXiv:cond-mat/0106144].

[33] M. E. J. Newman, "The structure and function of complex networks," SIAM Review 45 (2003): 167-256 [arXiv:cond-mat/0303516].

[34] R. van der Hofstad, Lecture Notes on Random Graphs and Complex Networks, available at http://www.win.tue.nl/ rhofstad (December 2010).

[35] Kurt Binder and Dieter W. Heermann, Monte Carlo Simulation in Statistical Physics: An Introduction, 5th ed. (New York: Springer, 2010).

[36] M. Abramowitz and I. A. Stegun, Handbook of Mathematical Functions (New York: Dover, 1965).

[37] I. Derényi, G. Palla, and T. Vicsek, "Clique percolation in random networks," Physical Review Letters 94 (2005): 160202 [arXiv:cond-mat/0504551].

[38] R. Cohen, K. Erez, D. ben-Avraham, and S. Havlin, "Resilience of the internet to random breakdowns," Physical Review Letters 85 (2000): 4626-4628 [arXiv:condmat/0007048]. 